\title{
Uterotonic drugs use for post partum hemorrhage: An audit of the third stage of labor management"
}

\author{
Etedafe P. Gharoro, Ehigha J. Enabudoso, Edafe E. Gharoro, Abieyuwa P. Osemwenkha
}

Department of Obstetrics and Gynecology, University of Benin Teaching Hospital, Benin City, Nigeria

Email: gharoro@uniben.edu, gharoro@hotmail.com

Received 31 March 2013; revised 21 April 2013; accepted 30 April 2013

Copyright (C) 2013 Etedafe P. Gharoro et al. This is an open access article distributed under the Creative Commons Attribution License, which permits unrestricted use, distribution, and reproduction in any medium, provided the original work is properly cited.

\section{ABSTRACT}

Objectives: Worldwide the use of uterotonic drugs has significantly reduced maternal mortality from postpartum hemorrhage. The objective is to audit the use of uterotonics in the active management of the third and fourth stages of labor. Methods: Personal data, diagnostic clinical information, blood loss and uterotonics administered were extracted from a cohort of 634 consecutive parturient. Trend in Shock Index (Pulse Rate/Systolic Blood Pressure) and 48 hours hematocrit changes were computed and analyzed. Results: There were 422 vagina deliveries and 212 caesarean sections. Primiparous mothers were 141 (34.2\%), while grand multiparous mothers were 14 (3.4\%). The mean visually estimated postpartum blood loss $165.9 \pm 80 \mathrm{ml}$. There was no significant difference in the mean blood loss between the three parity groups of parturient $[P=0.09]$. Fourteen parturient $(3.44 \%)$ had blood loss $\geq 500 \mathrm{ml}$. The value of Shock Index (Pulse Rate/Systolic Blood Pressure) in the study ranged between 0.43 and 1.38 . Logistic regression analysis of the variables associated with the switch between the three regimens of uterotonic drugs, showed a significant positive correlation between VEBL and uterotonic drugs administered [Pearson correlation $=0.130, P$-value $=0.008]$. In addition, there was a significant negative correlation between uterotonic drugs administered and Shock Index at 30 minutes and 2 hours postpartum. The correlation coefficient between VEBL and regimens of uterotonic drugs used was positive and significant $(P$ = 0.019). Conclusion: Visually estimated blood loss, with shock are the main Triggers involved in switching between uterotonic drugs regimens used in active management of PPH. Shock index calculation is vital

\footnotetext{
"Conflict of Interest notification: The authors wish to state that there is no conflict of interest in execution and publishing of this project. The authors privately funded the research.
}

in management decision. We advocate training of all birth attendants on VEBL.

Keywords: Post Partum Hemorrhage; Uterotonic Drugs; Visual Estimation of Blood Loss; Shock Index; Active Management of Third Stage of Labor

\section{INTRODUCTION}

Post partum hemorrhage (PPH) is the leading cause of maternal mortality worldwide [1-4]. The obstetric problem, $\mathrm{PPH}$, is underestimated because most delivery takes place outside hospital where no routine blood loss measurement is undertaken. The commonest cause of primary postpartum hemorrhage is uterine atony, which is poorly managed outside the main hospitals and most developing world countries [5-8].

Globally, prophylactic administration of uterotonic drugs in the active management of the third stage of labor has resulted in the reduction of severe morbidity and mortality from PPH. However, the uterotonic drugs used and the timing of administration of these drugs varies considerably, which may have significant implications on the reduction of post partum hemorrhage, the wellbeing of the both babies and mothers [9-11].

The third stage of labor is actively managed at the University of Benin Teaching Hospital (UBTH), with the use of uterotonic drugs according to an established departmental protocol.

1) Regimen $\mathrm{A}$ - An intravenous bolus of $0.25 \mathrm{mg} \mathrm{Er-}$ gometrine (or $10 \mathrm{IU}$ of Oxytocin if Ergometrine cannot be given) is administered at the delivery of the anterior shoulder followed by early clamping and cutting of the cord after the baby has been delivered. The placenta is then delivered by controlled cord traction without waiting for signs of placenta separation.

2) Regimen B-An intravenous bolus of $10 \mathrm{IU}$ of Oxytocin if Ergometrine cannot be given. Patients at increased risk of primary post partum hemorrhage have 40 
IU of Oxytocin added to $500 \mathrm{mls}$ of intravenous infusion to run over 4 to 6 hours to further maintain uterine contraction.

3) Regimen $\mathrm{C}$ - In the event of further vaginal bleeding or primary postpartum haemorrhage, $600 \mu \mathrm{g}$ of Misoprostol is inserted into the rectum or taken orally to maintain uterine contraction.

In the event of additional vaginal bleeding or anticipated $\mathrm{PPH}$, these uterotonic drugs are administered in various combinations, in addition to other measures, (e.g. uterine fundal massage and bladder catheterization) to establish uterine contraction and arrest hemorrhage.

Literatures on the audit and criteria for the switch in the use of these various uterotonic drugs regimens are scanty. In addition, there has been no recent audit of these departmental protocols and uterotonic drug regimens since introduction into the active management of the third stage of labor in the centre.

\section{OBJECTIVES}

To audit the use of uterotonics in the management of the third and fourth stages of labor for the control of primary post partum hemorrhage $\left(\mathrm{I}^{\mathrm{O}} \mathrm{PPH}\right)$. Specifically, to analyze the diagnostic information (the "Triggers") involved in the mental process of switching between the uterotonics regimens used in the active management of the third stage of labor and establish criteria for evaluating the suitability of patients transfer to the lying-in ward or referral.

\section{MATERIAL AND METHODS}

This was a prospective observational cohort study at the University of Benin Teaching hospital (UBTH), Benin City, between the months of April-July 2012 using pretested Data sheet to extract information at the time of delivery by a research assistant (Miss Iyore). Personal data and recorded clinical information like blood pressure (systolic and diastolic), pulse rate, respiratory rate, blood loss at delivery and uterotonics administered amongst others were extracted. Information collected with the data sheets was fed into the computer and analyzed using IBM SPSS Statistics version 19 software.

\section{Data Management}

Shock Index as a new variable was derived by calculation, from Pulse Rate divided by Systolic Blood Pressure $[$ Shock Index $=$ PR/Systolic BP]. Shock index at $30 \mathrm{~min}$ postpartum, 1 hour, 2 hours and 3 hours postpartum postpartum were calculated to derive trend. In addition Haematocrit change (48 hours postpartum) was computed according to (Gharoro and Enabudoso, 2009) [12]. Uterotonic drugs received by patients was recoded into a new variable according to the number of regimen out of the three in the departmental protocol a patient was given in the management of her third and fourth stages of labor.

Relationship between the uterotonic drug regimens and various observed labour management variables (PCV, Blood pressure, pulse rate, VEBL, Shock Index etc) was analysed by logistic regression (Pearson correlation coefficient) for any significant association. A P-value of $\leq 0.05$ was accepted as significant.

\section{RESULTS}

The study population was 634 consecutive parturient made up 422 vagina deliveries and 212 (33.4\%) Caesarean sections. Five parturient records were incomplete (three with no 48hrs post partum hematocrit and another two with no VEBL estimate). Primiparous patients were $141(34.2 \%)$, multipara $254(62.4 \%)$, while grandmultipara were $14(3.4 \%)$ of the total parturient (Table 1).

The mean age of mothers was $29.8 \pm 4.17$ years (range, 17 - 42 years). There was a significant difference in age between the three groups of mothers [ANOVAs $\mathrm{f}=$ 40.14, $\mathrm{P}=0.000]$. The mean postpartum blood loss (VEBL) of parturient was $165.94 \pm 80.03 \mathrm{ml}$ (Table 2).

There was no significant difference in the average blood loss between the three group of parturient [ANOVAs $\mathrm{f}=2.42, \mathrm{P}=0.09]$. However, there was a positive significant linear relationship between parity and blood loss [ANOVAs $\mathrm{f}=4.77, \mathrm{P}=0.03$ ]. Fourteen parturient $(3.4 \%)$ had blood loss equal to or greater than 500 $\mathrm{ml}$ in this study.

The value of Shock Index (Pulse Rate/Systolic Blood Pressure) in the study ranged between 0.43 and 1.38 . Logistic regression analysis of the variables associated

Table 1. Age distribution of parturient in the Study.

\begin{tabular}{cccccc}
\hline Parity & Mean & N (\% of Total N) & Std. Deviation & Min & Max \\
\hline Primip & 27.57 & $141(34.2 \%)$ & 3.66 & 17 & 40 \\
Multipara & 30.72 & $257(62.4 \%)$ & 3.90 & 20 & 41 \\
GrandMultip & 34.07 & $14(3.4 \%)$ & 4.14 & 27 & 42 \\
Total & 29.75 & $412(100.0 \%)$ & 4.17 & 17 & 42 \\
\hline
\end{tabular}

Table 2. Visual Estimate of blood loss amongst parturient.

\begin{tabular}{ccccc}
\hline Parity2 & Mean & Std. Deviation & Min & Max \\
\hline Primip & 177.66 & 81.60 & 100 & 700 \\
Multipara & 160.31 & 80.23 & 50 & 800 \\
GrandMultip & 150.00 & 35.36 & 100 & 200 \\
Total & 165.94 & 80.03 & 50 & 800 \\
\hline
\end{tabular}


with the switch between the three regimens of uterotonic drugs showed a significant positive correlation between VEBL and uterotonic drugs administered [Pearson correlation $=0.130, \mathrm{P}$-value $=0.008]$. Table 3 shows Pearson correlations test between VEBL in the study and some selected diagnostic clinical variables.

The Correlation test between uterotonic drugs use and shock index at 30 minutes and 2 hours postpartum was negative and significant [Pearson correlation $=-0.210$, P-value $=0.000$; and $-0.187,0.000]$. There was no significant correlation between uterotonic drugs used for postpartum management of hemorrhage and 48 hours hematocrit change. Table 4, shows that the correlation coefficient between VEBL and regimens of uterotonic drugs used for the active management of the third stage of labour was positive and significant $(\mathrm{P}=0.019)$.

\section{DISCUSSION}

In the active management of third stage of labor in UBTH, the first line uterotonic drugs (Ergometrine) is given at the delivery of the anterior shoulder. Subsequently, if there is a perceived threat of hemorrhage or there is brisk bleeding, the accoucheur takes additional steps to forestall potential blood loss. These steps involve switching between the three uterotonic regimen A, B or $\mathrm{C}$ in the departmental protocol for active management. The mental process which triggers the decision to administer the selected regimen (A or B or C) and the decision for further management need, require skills of different level and experience by birth attendants. The accoucheur is anxious to prevent postpartum hemorrhage. Visual stimulation by brisk blood loss and his visual estimation of the amount of blood already lost, play a significant role in this decision. Averting $\mathrm{I}^{\mathrm{O}} \mathrm{PPH}$ is essential to reducing maternal mortality, a key component of the WHO fifth millennium development goals (MDGs) [13-14].

This study revealed a significant positive correlation between blood loss and the switch between the uterotonic drugs regimens. Blood loss rate is a powerful visual

Table 3. Pearson Correlations between VEBL amongst parturient and selected clinical variables.

\begin{tabular}{|c|c|c|c|c|c|c|c|}
\hline & & VEBL & Uterotonic Drugs & Shock Index & Shock Index2 & $\begin{array}{l}\text { Haematocrit } \\
\text { Change }\end{array}$ & $\mathrm{PCV}$ \\
\hline \multirow{2}{*}{ VEBL } & Pearson Correlation & 1 & $0.130^{* *}$ & -0.015 & 0.072 & 0.071 & -0.081 \\
\hline & Sig. (2-tailed) & & 0.008 & 0.754 & 0.143 & 0.145 & 0.098 \\
\hline \multirow{2}{*}{ Uterotonic Drugs } & Pearson Correlation & $0.130^{* *}$ & 1 & $-0.210^{* *}$ & $-0.187^{* *}$ & 0.058 & -0.024 \\
\hline & Sig. (2-tailed) & 0.008 & & 0.000 & 0.000 & 0.234 & 0.622 \\
\hline \multirow{2}{*}{ Shock Index } & Pearson Correlation & -0.015 & $-0.210^{* *}$ & 1 & $0.252^{* *}$ & -0.029 & -0.035 \\
\hline & Sig. (2-tailed) & 0.754 & 0.000 & & 0.000 & 0.551 & 0.470 \\
\hline \multirow{2}{*}{$\begin{array}{c}\text { Shock Index@2 hours } \\
\text { Postpartum }\end{array}$} & Pearson Correlation & 0.072 & $-0.187^{* *}$ & $0.252^{* *}$ & 1 & 0.031 & -0.010 \\
\hline & Sig. (2-tailed) & 0.143 & 0.000 & 0.000 & & 0.527 & 0.837 \\
\hline \multirow{2}{*}{ Haematocrit Change } & Pearson Correlation & 0.071 & 0.058 & -0.029 & 0.031 & 1 & $0.453^{* *}$ \\
\hline & Sig. (2-tailed) & 0.145 & 0.234 & 0.551 & 0.527 & & 0.000 \\
\hline \multirow{2}{*}{ PCV } & Pearson Correlation & -0.081 & -0.024 & -0.035 & -0.010 & $0.453^{* *}$ & 1 \\
\hline & Sig. (2-tailed) & 0.098 & 0.622 & 0.470 & 0.837 & 0.000 & \\
\hline
\end{tabular}

${ }^{* *}$ Correlation is significant at the 0.01 level (2-tailed).

Table 4. Correlation coefficients between VEBL amongst parturient and selected clinical variables.

\begin{tabular}{|c|c|c|c|c|c|c|}
\hline & \multirow{2}{*}{ Model } & \multicolumn{2}{|c|}{ Unstandardized Coefficients } & \multirow{2}{*}{$\begin{array}{c}\text { Standardized Coefficients } \\
\text { Beta }\end{array}$} & \multirow{2}{*}{$\mathrm{t}$} & \multirow{2}{*}{ Sig. } \\
\hline & & B & Std. Error & & & \\
\hline \multirow{5}{*}{1} & (Constant) & 87.778 & 37.402 & & 2.347 & 0.019 \\
\hline & Uterotonic Drugs & 12.504 & 4.385 & 0.143 & 2.852 & 0.005 \\
\hline & Shock Index & -5.631 & 33.342 & -0.009 & -0.169 & 0.866 \\
\hline & Shock Index 2 & 86.558 & 44.334 & 0.099 & 1.952 & 0.052 \\
\hline & Haematocrit Change & 1.266 & 1.031 & 0.060 & 1.228 & 0.220 \\
\hline
\end{tabular}

Dependent Variable: VEBL. 
stimulant (a "trigger") for identifying potential danger, this stimulus is re-enforced by the actual volume of the blood lost.

Unfortunately, the absolute volume (quantity) of blood lost may not be known immediately by the accoucheur. He therefore relies on an estimate of the amount of blood loss (VEBL) to make a management decision to switch between the different uterotonic regimens. Visual estimation of blood loss is notorious for its inaccuracy [15, 16] however, a previous study from Benin [12] and other workers [17-20] have shown that this process (VEBL) can be improved by training, especially if a "reference volume" or quantification method is used to simulate the amount of blood spilled. In addition, there is a good correlation between VEBL and 48 hours postpartum hematocrit change [12]. Improving the ability of the accoucheur to accurately estimate blood loss by VEBL method needs training and retraining to reduce human error in quantification [21].

This skill once acquired becomes a useful tool to reduce postpartum hemorrhage and by implication maternal mortality.

Vital signs monitoring compliment VEBL in active management of the third stage of labor to prevent postpartum hemorrhage. The accoucheur does not rely absolutely on VEBL to switch between regimens. Most published literature compare drugs used for the control of PPH [22,23]. Worldwide, literatures on when to switch from a resistant or failed therapeutic method to another are few. Shock plays an important role in his decision making process. Irita and Inada (2010) have evaluated the usefulness of trend in shock index in the management of obstetric patients' need for blood transfusion [24]. Results from this study showed that there was a significant negative correlation between shock index at $30 \mathrm{~min}$ and two hours and the use of uterotonic drugs regimens. Trend in shock index plays a role in the decision making process for additional resuscitation and the need for blood transfusion. A randomized prospective study may be necessary to develop a scoring system to evaluate further, the place of shock index in actively managing parturient.

Other predicative variables found to be of clinical significance in the decision making process during the active management of the third stage of labor in this study were diastolic blood pressure and weight. There was no significant correlation between the use of uterotonic drugs and age of parturient.

\subsection{Conclusion}

Visually estimated blood loss, with shock are the main Triggers involved in switching uterotonic drugs regimens used in active management of PPH. Shock index calcula- tion is vital in management decision.

\subsection{Recommendations}

We advocate all birth attendants nationally should be trained on Visual estimation of blood loss. A reference volume of $350 \mathrm{ml}$ (one standards Coca-Cola bottle) should be used to simulate the Volume of blood loss during training exercises.

Shock index should be computed with each vital sign recorded for the parturient during management of the third stage of labor and post partum until transfer to the lying-in-ward or referral to a tertiary health institution.

\section{ACKNOWLEDGEMENTS}

The authors wish to acknowledge Miss Iyore Osayande, Data Processing Officers in the department of Obstetrics and Gynecology UBTH, for her role in collecting and collating the data even at odd hours of the day. We also want to declare that there is no conflict of interest in the research work and the authors received no material or financial assistance from any donor agency or organizations; private funds were used for the research.

\section{REFERENCES}

[1] Wise, A. and Clark, V. (2010) Challenges of major obstetric hemorrhage. Best Practice \& Research Clinical Obstetrics \& Gynaecology, 24, 353-365. doi:10.1016/j.bpobgyn.2009.11.011

[2] Selo-Ojeme, D.O. (2002) Primary postpartum hemorrhage. Journal of Obstetrics \& Gynaecology, 22, 463-469. doi:10.1080/0144361021000003555

[3] World Health Organization (2009) WHO guidelines for the management of postpartum hemorrhage and retained placenta.

www.who.int/entity/pmnch/topics/maternal/2009 who97 89241598514/en/

[4] Knight, M., Callaghan, W.M., Berg, C., Alexander, S., Bouvier-Colle, M.H., Ford, J.B., Joseph, K.S., Lewis, G., Liston, R.M., Roberts, C.L., Oats, J. and Walker, J. (2009) Trends in postpartum hemorrhage in high resource countries: A review and recommendations from the International Postpartum Hemorrhage Collaborative Group. BMC Pregnancy Childbirth, 9, 55. doi:10.1186/1471-2393-9-55

[5] Al-Zirqi, I., Vangen, S., Forsen, L. and Stray-Pedersen, B. (2008) Prevalence and risk factors of severe obstetric hemorrhage. BJOG: An International Journal of Obstetrics \& Gynaecology, 115, 1265-1272. doi:10.1111/j.1471-0528.2008.01859.x

[6] Leduc, D., Senikas, V., Lalonde, A.B., Ballerman, C., Biringer, A., Delaney, M., Duperron, L., Girard, I., Jones, D., Lee, L.S., Shepherd, D. and Wilson, K. (2009) Active management of the third stage of labor: Prevention and treatment of postpartum hemorrhage. Journal of Obstetrics and Gynaecology Canada, 31, 980-993.

[7] Ijaiya, M.A., Aboyeji, A.P. and Abubakar, D. (2003) 
Analysis of 348 consecutive cases of primary postpartum hemorrhage at a tertiary hospital in Nigeria. Journal of Obstetrics \& Gynaecology, 23, 374-377. doi:10.1080/0144361031000119529

[8] Bibi, S., Danish, N., Fawad, A. and Jamil, M. (2007) An audit of primary post partum hemorrhage. Journal of Ayub Medical College Abbottabad, 19, 102-106.

[9] Ajenifuja, K.O., Adepiti, C.A. and Ogunniyi, S.O. (2010) Post partum hemorrhage in a teaching hospital in Nigeria: A 5-year experience. African Health Sciences, 10, 71-74.

[10] Soltani, H., Hutchon, D.R. and Poulose, T.A. (2010) Timing of prophylactic uterotonics for the third stage of labor after vaginal birth. Cochrane Database of Systematic Reviews, 8, Article ID: CD006173.

[11] Begley, C.M., Gyte, G.M., Devane, D., McGuire, W. and Weeks, A. (2011) Active versus expectant management for women in the third stage of labor. Cochrane Database of Systematic Reviews, 11, Article ID: CD007412.

[12] Gharoro, E.P. and Enabudoso, E.J. (2009) Relationship between visually estimated blood loss at delivery and postpartum change in hematocrit. Journal of Obstetrics and Gynecology, 29, 517-520. doi:10.1080/01443610903003159

[13] Cohain, J.S. (2010) The dangers of planned hospital births. Midwifery Today with International Midwife, 94, 30-32, 67-68.

[14] WHO (2012) Millennium Development Goals (MDGs). www.who.int/entity/mediacentre/factsheets/fs290/en/

[15] Patel, A., Goudar, S.S., Geller, S.E., Kodkany, B.S., Edlavitch, S.A., Wagh, K., Patted, S.S., Naik, V.A., Moss, N. and Derman, R.J. (2006) Drape estimation vs. visual assessment for estimating postpartum hemorrhage. International Journal of Gynecology \& Obstetrics, 93, 220-224. doi:10.1016/j.ijgo.2006.02.014

[16] Razvi, K., Chua, S., Arulkumaran, S. and Ratnam, S.S. (1996) A comparison between visual estimation and laboratory determination of blood loss during the third stage of labor. Australian and New Zealand Journal of Obstetrics and Gynaecology, 36, 152-154. doi:10.1111/j.1479-828X.1996.tb03273.x
[17] Dildy 3rd, G.A., Paine, A.R., George, N.C. and Velasco, C. (2004) Estimating blood loss: Can teaching significantly improve visual estimation? Obstetrics \& Gynecology, 104, 601-606. doi:10.1097/01.AOG.0000137873.07820.34

[18] Toledo, P., McCarthy, R.J., Burke, C.A., Goetz, K., Wong, C.A. and Grobman, W.A. (2010) The effect of live and web-based education on the accuracy of bloodloss estimation in simulated obstetric scenarios. American Journal of Obstetrics and Gynecology, 202, 400.e1-400.e5.

[19] Yoong, W., Karavolos, S., Damodaram, M., Madgwick, K., Milestone, N., Al-Habib, A., Fakokunde, A. and Okolo, S. (2010) Observer accuracy and reproducibility of visual estimation of blood loss in obstetrics: How accurate and consistent are health-care professionals? Archives of Gynecology and Obstetrics, 281, 207-213. doi:10.1007/s00404-009-1099-8

[20] Sukprasert, M., Choktanasiri, W., Ayudhya, N.I., Promsonthi, P. and O-Prasertsawat, P. (2006) Increase accuracy of visual estimation of blood loss from education programme. Journal of the Medical Association of Thailand, 89, S54-S59.

[21] Buckland, S.S. and Homer, C.S. (2007) Estimating blood loss after birth: Using simulated clinical examples. Women and Birth, 20, 85-88. doi:10.1016/j.wombi.2007.01.001

[22] Joy, S.D., Sanchez-Ramos, L. and Kaunitz, A.M. (2003) Misoprostol use during the third stage of labor. International Journal of Gynecology \& Obstetrics, 82, 143-152. doi:10.1016/S0020-7292(03)00146-2

[23] Stanton, C.K., Newton, S., Mullany, L.C., Cofie, P., Agyemang, C.T., Adiibokah, E., Darcy, N., Khan, S., Levisay, A., Gyapong, J., Armbruster, D. and OwusuAgyei, S. (2012) Impact on postpartum hemorrhage of prophylactic administration of oxytocin 10 IU via Uniject ${ }^{\mathrm{TM}}$ by peripheral health care providers at home births: Design of a community-based cluster-randomized trial. BMC Pregnancy \& Childbirth, 12, 42. doi:10.1186/1471-2393-12-42

[24] Irita, K. and Inada, E. (2011) Guideline for management of critical bleeding in obstetrics. Masui, 60, 14-22.

\author{
Abbreviations and Acronyms \\ VEBL-Visual Estimation blood Loss \\ UBTH-University of Benin Teaching Hospital \\ PPH_Postpartum Hemorrhage
}

$\mathrm{I}^{\mathrm{O}} \mathrm{PPH}$-Primary Postpartum Hemorrhage

$\mathrm{Mg}$ - milligram

IU-International Unit

$\mu \mathrm{g}-$ Microgram 